Vania Modesto-Lowe, MD, MPH

University of Connecticut School of Medicine,

Farmington, CT; Quinnipiac University, Hamden, CT:

Connecticut Valley Hospital, Middletown, CT
Agata K. Harabasz, MD

University of Connecticut School of Medicine,

Farmington, CT; Middlesex Health Family Medicine,

Middletown, CT
Sophia A. Walker, MD

University of Connecticut School of Medicine,

Farmington, CT

\title{
Quetiapine for primary insomnia: Consider the risks
}

\section{ABSTRACT}

The second-generation antipsychotic drug quetiapine (Seroquel) is increasingly being used off-label for treating insomnia in the general population, possibly to avoid standard medications with known addictive qualities and adverse side effects. However, evidence to support using it in this way is scant, and quetiapine is associated with weight gain and other metabolic effects. It must be used cautiously and with appropriate monitoring for adverse effects and abuse.

\section{KEY POINTS}

Quetiapine affects multiple central nervous system receptors, resulting in a variety of effects, including sedation.

The use of quetiapine to treat insomnia should be confined primarily to patients with comorbid mood or schizophrenia spectrum disorders.

Compared with many other antipsychotic drugs, quetiapine is less associated with dystonia and extrapyramidal side effects but tends to cause weight gain, metabolic syndrome, and QTc prolongation.

Body mass index, weight, blood pressure, fasting glucose, and lipid levels should be measured before starting treatment and then regularly monitored, even for low doses.

Despite having no euphoric effects, quetiapine is often abused to enhance or counter side effects of illicit drugs.

Quetiapine carries particular risks for elderly patients.
DESCRIPTIONS FOR QUeTIAPINE (Seroquel), a second-generation antipsychotic medication, have risen sharply in recent years. ${ }^{1,2}$ Despite its approval by the US Food and Drug Administration (FDA) only for the treatment of schizophrenia, bipolar disorder (depression, acute mania, and maintenance), and major depressive disorder (as an adjunct medication), ${ }^{3}$ only a minority of patients filling prescriptions for quetiapine have these diagnoses. Rather, quetiapine is increasingly being used off-label, including for insomnia, anxiety, agitation, and posttraumatic stress disorder (PTSD). ${ }^{4}$ It is generally regarded as being nonaddictive and having a good safety profile. However, its cardiometabolic effects and potential for abuse warrant caution for its off-label use.

This article focuses on the use of quetiapine for treating insomnia, its basic pharmacology, evidence of efficacy, and adverse effects, and it provides recommendations for clinical monitoring of patients receiving the drug.

\section{AN INCREASINGLY POPULAR DRUG}

Second-generation antipsychotic use has increased worldwide, with quetiapine, risperidone, and olanzapine being the most frequently prescribed. ${ }^{1}$ In Canada, prescriptions written by family physicians for quetiapine increased 300\% from 2005 to 2012, with a 10. fold increase in its use for sleep disorders. ${ }^{2}$ The pattern was similar in the United States from 1996 to 2003, with up to $70 \%$ of prescriptions for second-generation antipsychotics being written for conditions other than psychosis. ${ }^{4}$

Bertisch et al, ${ }^{5}$ using US National Health and Nutrition Examination Survey data from 1999 to 2010, found that nearly 3\% of 32,328 respondents reported having used a common- 


\section{TABLE 1}

\section{Quetiapine activity in the central nervous system}

\begin{tabular}{l|c|c|c|c}
\hline Doses & \multicolumn{2}{|c|}{ Low (25-100 mg daily) } & Medium (300-600 mg daily) & High (> 800 mg daily) \\
\hline Receptors & Histamine 1 & $\begin{array}{c}\text { Alpha 1/alpha 2 } \\
\text { adrenergic }\end{array}$ & $\begin{array}{c}\text { 5-HT1A, 5-HT2A/B, } \\
\text { 5-HT2C }\end{array}$ & D2 \\
\hline Activity & Antihistaminergic & Antiadrenergic & Serotonergic & Antidopaminergic \\
\hline Effect & \multicolumn{2}{|c|}{ Sedation } & Antidepressant properties & Antipsychotic properties \\
\hline
\end{tabular}

ly prescribed insomnia medication over the previous month. Quetiapine ranked fourth among frequently prescribed medications, following the "Z-drugs" (the benzodiazepine receptor agonists zolpidem, zaleplon, and eszopiclone), trazodone, and benzodiazepines. ${ }^{5}$

Prescriptions for quetiapine to treat sleep disturbances have also increased in Australia, New Zealand, and the United Kingdom. ${ }^{6-8}$

\section{POTENTIAL FOR ABUSE}

Quetiapine, used alone, does not produce euphoria or other pleasurable effects typically associated with drugs of abuse, but it can enhance or counter the side effects of substances such as marijuana, cocaine, and heroin, ${ }^{9}$ a practice that is colloquially referred to as "seroquelling." Although the mechanism underlying its rewarding effects remains elusive, misuse of the drug may be driven by its ability to counteract overstimulation caused by other substances of abuse. ${ }^{10}$ There are also reports of quetiapine use for "self-detoxification," in an effort to mitigate withdrawal symptoms from other substances such as alcohol, cocaine, benzodiazepines, and opioids. ${ }^{9}$ These trends appear to be more prevalent in correctional facilities (where commonly abused drugs are not readily available) and among patients with a previous history of substance abuse. ${ }^{9}$ Those who abuse quetiapine typically consume high doses, which has led to several cases of accidental overdose, some of which have been fatal. ${ }^{9}$

These emerging patterns of abuse, along with the drug's potential metabolic and cardiac complications, signal the need for caution for use in the general patient population.

\section{DIFFERENT DOSES}

\section{HAVE DIFFERENT EFFECTS}

Quetiapine works similarly to other secondgeneration antipsychotics, but its uniqueness lies in the various affinities of the drug and its active metabolite (norquetiapine) for multiple central nervous system receptors in a dose-dependent manner (Table 1). Lower dosages primarily affect histaminergic (H1) and alpha 1 and alpha 2 adrenergic receptors, mediating sedative effects. ${ }^{11}$ Medium and high doses have an additive affinity for serotonergic receptors $(5-\mathrm{HT} 1 \mathrm{~A}, 5-\mathrm{HT} 2 \mathrm{~A}, 5-\mathrm{HT} 2 \mathrm{~B}$, 5-HT2C) and the dopamine D2 receptors, causing mood stabilization and improvements in anxiety, deep sleep, and psychosis. ${ }^{12}$

This quality of increasing doses causing different effects has led to the "Goldilocks" analogy: "Papa Bear" doses (> 800 mg daily) are for treatment of schizophrenia, "Mama Bear" doses (300-600 mg daily) are for mood disorders, and "Baby Bear" doses (25-100 mg daily) are for sedative-hypnotic effects. ${ }^{13}$

Among antipsychotics drugs, quetiapine is the least potent binder of the D2 receptor and has the fastest dissociation time from it, which explains the larger doses required for achieving antipsychotic effects as well as its lower incidence of extrapyramidal side effects. ${ }^{12,14}$

\section{PHARMACOKINETIC PROFILE LENDS ITSELF TO ABUSE}

Quetiapine is available as an oral tablet in an immediate-release formulation (time to peak plasma level 1.5 hours) and an extendedrelease formulation (6 hours)., ${ }^{2,15}$ Its half-life, about 6 hours, is the shortest of all the secondgeneration antipsychotics.

\section{Cardiometabolic effects and potential for abuse warrant caution for its off-label use}


The short duration to peak plasma concentration with the immediate-release formulation is generally comparable with that of many of the approved hypnotics, including Zdrugs and benzodiazepines. ${ }^{16}$ At peak plasma levels, H1-receptor occupancy is more than $90 \%$ at just $50 \mathrm{mg}$ of the immediate-release formulation, ${ }^{13}$ which is consistent with the receptor profile for strong hypnotic effects even at the lowest therapeutic doses.

Quetiapine's pharmacokinetic profile may make it more attractive for abuse, especially for crushing tablets for intravenous injection or intranasal snorting. It can also be mixed with other drugs of abuse ${ }^{9}$ to achieve faster and more intense effects, such as sedation and relaxation. Quetiapine taken through such alternative routes is associated with an increased risk of neuroleptic toxicity. ${ }^{10}$

\section{ADVERSE EFFECTS}

\section{Weight gain, metabolic effects}

Common adverse effects of second-generation antipsychotics include weight gain and motor symptoms. ${ }^{17}$ Clozapine and olanzapine are more recognized for causing weight gain, but

Use of secondgeneration antipsychotics has increased worldwide long-term use of quetiapine is also associated with moderate weight gain (10 kg on average), as well as development of metabolic syndrome. ${ }^{18}$ The mechanism behind weight gain in this class of drugs is unclear, but antihistaminergic effects may be causing enhanced appetite.

Second-generation antipsychotics also increase levels of blood glucose and low-density lipoprotein cholesterol, effects that seem to resolve when treatment is stopped. ${ }^{19,20}$ While weight gain alone is concerning, the metabolic changes associated with second-generation antipsychotic use can lead to higher risk of cardiovascular disease and stroke, with quetiapine among the antipsychotic drugs associated with the largest metabolic effects. ${ }^{21}$

\section{Low doses not totally harmless}

Whether quetiapine's metabolic effects occur even at low doses has been investigated in retrospective studies. Cates et $\mathrm{al}^{22}$ studied 43 patients taking low-dose quetiapine for insomnia. About two-thirds of them gained weight: daily dosages below $200 \mathrm{mg}$ at bedtime used for an average of 11 months were associated with an average weight gain of $4.9 \mathrm{lb}(P=$ $.037)$ and a body mass index (BMI) increase of $0.8 \mathrm{~kg} / \mathrm{m}^{2}(P=.048)$.

Carr et $\mathrm{al}^{23}$ studied 403 veterans taking lowdose quetiapine (average daily dose $116.8 \mathrm{mg}$ ) for an average of 44 months. Statistically significant increases were found in systolic blood pressure $(1.95 \mathrm{~mm} \mathrm{Hg}, P=.036)$, diastolic blood pressure $(1.97 \mathrm{~mm} \mathrm{Hg}, P=.001), \mathrm{BMI}(0.52 \mathrm{~kg} /$ $\left.\mathrm{m}^{2}, P=.001\right)$, weight $(1.88 \mathrm{~kg}, P=.002)$, and fasting blood glucose $(6.71 \mathrm{mg} / \mathrm{dL}, P=.002)$.

Williams et al ${ }^{24}$ investigated low-dose quetiapine ( $<100 \mathrm{mg}$ daily for at least 1 month) in 534 patients in military hospitals. The mean weight gain was $5.56 \pm 1.25 \mathrm{lb}(P<.001)$ at 6 months and $10.58 \pm 2.20 \mathrm{lb}(P<.001)$ at 12 months compared with baseline.

\section{Extrapyramidal effects}

The low affinity of quetiapine for the D2 receptor, as well as a preference for binding to D2 receptors in the limbic pathway over the striatum, make movement disorders a less prominent side effect. ${ }^{25}$ However, extrapyramidal adverse effects do occur, ${ }^{19}$ with reports of restless legs syndrome, tardive dyskinesia, akathisia, and periodic leg movement disorder. ${ }^{14}$ In studies of quetiapine for bipolar disorder, the incidence of extrapyramidal symptoms increased in a dose-dependent manner, occurring in around $7 \%$ to $12 \%$ of patients. ${ }^{26,27}$

\section{QTc prolongation}

Quetiapine's labeling carries a warning for QTc prolongation. Risk is dose-dependent.

\section{Sedation}

Given their action on histamine receptors, second-generation antipsychotics commonly cause sedation. Quetiapine also has sleep latency-enhancing properties (reducing the time from being fully awake to falling asleep), attributable to its serotonergic action, leading to the drug's off-label use for insomnia. ${ }^{11}$ Outside of this context, sedation is generally considered to be an undesirable side effect for most patients. ${ }^{18}$

\section{Some side effects lessened}

Quetiapine's low affinity for D2 receptors results in fewer of the endocrine side effects associated with antipsychotic drugs, namely, prolactin elevation and associated amenor- 
rhea, galactorrhea, sexual dysfunction, and osteoporosis. . $^{12,14,18}$

\section{CONCERNS FOR THE ELDERLY}

Use of second-generation antipsychotics is becoming more widespread in the elderly as clinicians try to avoid adverse effects (particularly extrapyramidal symptoms) of traditional antipsychotics. ${ }^{28}$ However, use of second-generation antipsychotics comes with its own set of risks in this population. Second-generation antipsychotics have received FDA black-box warnings for a nearly twofold increase in incidence of cardiovascular events, stroke, and overall mortality. ${ }^{21}$ The American Geriatrics Society 2019 Beers Criteria note an increased rate of cognitive decline associated with antipsychotics in patients with dementia and strongly recommends avoiding their use in this population. ${ }^{29}$

Given its action on adrenergic receptors, quetiapine can be associated with orthostatic hypotension, especially in the elderly. ${ }^{12}$ Syncope from hypotension can lead to hip fractures, transient ischemic attacks, myocardial infarction, and even death. ${ }^{21}$ A 2013 study investigating 4 second-generation antipsychotics to treat psychiatric conditions in patients over age 40 found a high incidence of side effects (50\% of participants) and life-threatening conditions (24\%), noting that adverse effects were twice as common with quetiapine than with the other drugs. ${ }^{30}$ Furthermore, quetiapine's clearance is about $40 \%$ lower in elderly patients than in younger patients. ${ }^{25} \mathrm{In}$ view of the increasing use of second-generation antipsychotics in the elderly, these findings are especially worrisome.

\section{STANDARD INSOMNIA MEDICATIONS HAVE DISADVANTAGES}

The Diagnostic and Statistical Manual of Mental Disorders, fifth edition ${ }^{31}$ defines insomnia as difficulty initiating sleep, difficulty maintaining sleep, or early morning awakening, resulting in significant physical or emotional distress. Insomnia affects about $30 \%$ of the population worldwide, and $10 \%$ have symptoms that severely affect their daily function. ${ }^{32}$ People with insomnia often experience irritability, fatigue, physical distress, and impaired cognition. ${ }^{32}$ The disorder has been linked with poor academic and work functioning as well as cardiovascular disease, cancer, diabetes, and hypertension. ${ }^{32}$ Therapeutic goals for patients with insomnia include improving sleep and daytime function and reducing distress. ${ }^{32}$

\section{Benzodiazepines and Z-drugs}

Benzodiazepines and Z-drugs have been approved by the FDA for treating insomnia. They enhance gamma-aminobutyric acid (GABA) neurotransmission, leading to sedation, reduced sleep latency, and increased sleep efficiency. While benzodiazepines nonspecifically bind to GABA receptors in the central nervous system, Z-drugs preferentially bind to the GABA receptor alpha-1 subunit, making them less sedating. This subunit mediates sleep effects while appearing to confer a better safety profile for the Z-drugs. ${ }^{11}$ However, to varying degrees, both classes are associated with cognitive and memory impairments, rebound insomnia, risk of dependence and misuse, as well as car accidents, falls, and workplace accidents. ${ }^{11}$

Because benzodiazepines are associated with tolerance and subsequent withdrawal, treatment is recommended for only 2 to 4 weeks at a time. ${ }^{33}$ Given these risks and limitations, as well as the status of these drugs as schedule IV controlled substances requiring prescription monitoring, physicians are often hesitant to prescribe them to patients in favor of alternatives. ${ }^{6}$

\section{IS QUETIAPINE THE ANSWER?}

Quetiapine is increasingly being turned to as an off-label alternative for treating insomnia, owing to its well-known sedative and sleeppromoting effects at low doses.

\section{Study in healthy men}

In a seminal 2004 study, Cohrs et $\mathrm{al}^{34}$ explored the effects of quetiapine on sleep architecture and subjective sleep quality in 14 healthy men, using self-assessment and polysomnography recordings 3 times (4 days apart) for 3 consecutive nights. In the second set (standard sleep conditions) and third set (acoustic stress conditions), treatment was given on the first and second night, consisting of either placebo, quetiapine $25 \mathrm{mg}$, or quetiapine $100 \mathrm{mg}$ by mouth 1 hour before sleep.
Secondgeneration antipsychotics increase weight, glucose, and low-density lipoprotein cholesterol 
Relative to placebo, quetiapine $25 \mathrm{mg}$ and $100 \mathrm{mg}$ significantly improved subjective sleep quality and sleep initiation, duration, and efficiency, with a dose-dependent increase in stage 2 sleep. Periodic leg movements during sleep were noted with quetiapine $100 \mathrm{mg}$.

\section{Studies in mood and psychotic disorders} Guidelines from the American Academy of Sleep Medicine ${ }^{35}$ and others agree that offlabel use of antipsychotics should be avoided, although quetiapine may be useful for insomnia in patients with psychiatric disorders.

In 2009 , Wine et $\mathrm{al}^{36}$ reviewed 10 controlled studies and case reports assessing the effects of immediate-release quetiapine on sleep in patients with bipolar disorder, schizophrenia, history of trauma, or depression. Their analysis suggested that quetiapine improved total sleep time, efficiency, and subjective sleep within a dose range of $12.5 \mathrm{mg}$ to 800 mg; however, decreased rapid-eye-movement sleep was noted in some populations. Notable adverse events included akathisia, metabolic changes, and periodic leg movements.

In 2014, Anderson and Vande Griend ${ }^{37}$ analyzed studies investigating quetiapine for insomnia, including a review of the 2010 EMBOLDEN II (Efficacy of Seroquel for Bipolar Depression) trial, ${ }^{38}$ which found that quetiapine improved sleep in patients with bipolar depression over 8 weeks. Despite this, Anderson and Vande Griend argued that given the insufficient evidence of efficacy for treating insomnia and potential risks associated with the drug, they did not recommend quetiapine for insomnia even in patients with psychiatric disorders.

In 2008, Endicott et al ${ }^{39}$ conducted a secondary analysis of the large multicenter, randomized, placebo-controlled BOLDER (Bipolar Depression) I and II trials, which had demonstrated efficacy of quetiapine for acute bipolar depression. ${ }^{26,27}$ In a secondary analysis, they found sleep quality and total sleep time to be improved among patients treated with quetiapine.

\section{Studies in posttraumatic stress disorder}

Some data support the use of quetiapine for PTSD symptoms such as nightmares and insomnia. A 2005 study $^{40}$ in 20 postwar veterans with PTSD found that adding low-dose quetiapine resulted in significant improvements in sleep quality, latency, duration, night ter- rors, and nightmares.

A 2016 randomized controlled trial ${ }^{41}$ investigating quetiapine monotherapy for treatment of PTSD found a statistically significant reduction in the total Clinician-Administered PTSD Scale score $(P=.02)$ and its re-experiencing $(P=.0004)$ and hyperarousal $(P=$ .007) subscale scores compared with placebo. No statistically significant differences in weight or blood pressure were found between groups, but quetiapine use was associated with increased somnolence and sedation.

Byers et $\mathrm{al}^{42}$ compared prazosin and quetiapine for treating nighttime symptoms in veterans with PTSD $(\mathrm{N}=237)$ and found that shortterm effectiveness of the 2 drugs was similar at $60 \%$. However, patients taking prazosin were significantly more likely to remain in the study, and those in the quetiapine group were likelier to stop the medication because of side effects.

\section{Studies for primary insomnia}

A 2012 summary of a 2011 Agency for Healthcare Research and Quality review of safety and efficacy data of off-label uses for atypical antipsychotics ${ }^{17}$ was inconclusive concerning the use of quetiapine for insomnia. Only 1 relevant study met the authors' inclusion criteria: a 2010 study $^{43}$ in just 13 patients with primary insomnia conducted in Thailand. In this randomized, double-blinded, placebo-controlled trial, participants received either quetiapine $25 \mathrm{mg}$ or placebo each night for 2 weeks. There were nonsignificant trends for longer total sleep time and shorter sleep latency in the quetiapine group.

The 2014 Anderson and Vande Griend ${ }^{37}$ analysis of studies investigating quetiapine for insomnia concluded that data were insufficient to make a decision regarding safety and efficacy for this use. Existing literature was scarce, consisting of studies that included only small numbers of patients with specific conditions, and few studies used objective sleep quality measures such as polysomnography. They concluded that given its high side-effect profile and lack of data on efficacy, quetiapine should not be used to treat insomnia.

In 2018, Atkin et $\mathrm{al}^{11}$ reviewed the evidence for multiple pharmacologic agents used for insomnia and compared their effect on sleep physiology. The authors concluded that there was 
limited evidence to support the use of quetiapine for insomnia in the general population, but there may be a role for using it to improve sleep in patients with conditions that can be treated with quetiapine, such as psychotic or mood disorders. They also concluded that compared with other medications used for insomnia, quetiapine poses a low risk of dependence.

\section{MANY BELIEVE OFF-LABEL QUETIAPINE TO BE SAFER THAN ALTERNATIVES}

Why do some clinicians prescribe quetiapine off-label?

In 2017, Chow et a ${ }^{44}$ reviewed the use of nighttime-only quetiapine in 83 children and adolescents treated in an inpatient psychiatric setting. Forty seven $(57 \%)$ received it for insomnia alone, and 21 (25\%) received it for insomnia plus another indication. Youths in the first group had longer lengths of stay and were more likely to be female and have anxiety, eating disorders, or borderline personality disorder. Hence, quetiapine probably was prescribed in an effort to target multiple issues (eg, mood, anxiety, sleep) while avoiding polypharmacy.

In 2018, Kelly et $\mathrm{al}^{45}$ investigated outpatient prescribing of quetiapine among family physicians. Quetiapine was generally reserved for patients who had not responded to other therapies or had psychiatric comorbidities or difficult social backgrounds. Many physicians prescribed it to avoid benzodiazepine use and minimize risk for abuse. Many physicians interviewed believed that low doses of quetiapine were generally safe, so they did not monitor patients for side effects.

\section{CLINICAL RECOMMENDATIONS}

\section{Considerations for use for primary insomnia}

Given the scant evidence in favor of using quetiapine in the general population to treat insomnia and the risk of metabolic side effects even at low doses, the drug should be used with caution and only after other drug options have been exhausted. Practitioners should also consider prescribing it only in short courses in an effort to limit its long-term effects. ${ }^{20}$ For patients currently using it, providers should look for opportunities to discontinue it if clinically indicated.
TABLE 2

\section{Recommendations for monitoring during quetiapine treatment}

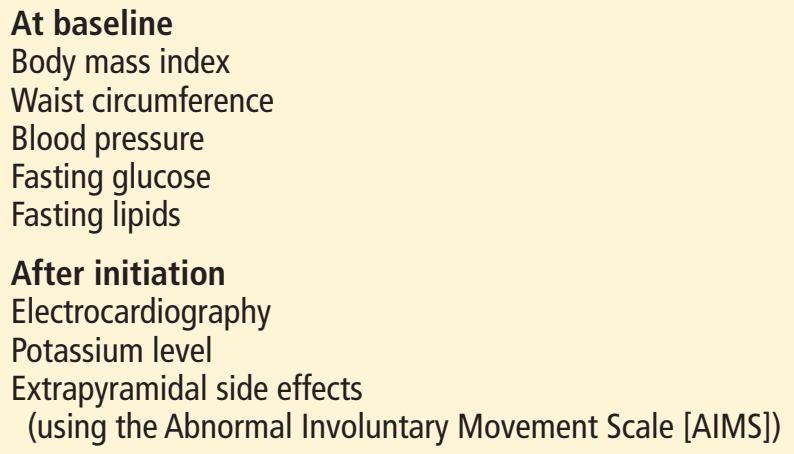

After initiation

Electrocardiography

Potassium level

Extrapyramidal side effects

(using the Abnormal Involuntary Movement Scale [AIMS])

At 4,8 , and 12 weeks after initiation

Body mass index

Three months after initiation

Blood pressure

Fasting glucose

Fasting lipids

Routinely at follow-up visits

Body mass index

Extrapyramidal side effects (using AIMS)

Signs of misuse, abuse, or other drug-seeking behavior

\section{Annually}

Blood pressure

Fasting glucose

Fasting lipids

With dose or risk factor changes

Electrocardiography

Potassium level

Based on information in reference 20.

Before prescribing quetiapine, one should compare its expected benefits (improved mood, sleep, functioning) and risks (metabolic syndrome, motor side effects, abuse). ${ }^{21}$

Age and comorbidities should be considered, particularly personal and family history of obesity, diabetes, dyslipidemia, hypertension, and cardiovascular disease. ${ }^{20}$

Given its reduced drug clearance in older individuals, caution should be used when dosing quetiapine for patients over age 65.

Patients at risk of QTc prolongation (those with heart disease, advanced age, taking other QTc-prolonging medications, electrolyte abnormalities, bradycardia, con- 
genital long QT syndrome) should have an electrocardiogram and potassium level obtained after initiation and any time the dose or risk factors change. ${ }^{21}$

As with other second-generation antipsychotic drugs, signs of agranulocytosis should be monitored.

In pregnancy, antipsychotics may increase the risks of gestational diabetes, hypertension, and congenital malformation, although evidence for the safety of quetiapine in this setting is limited and conflicting. ${ }^{21}$

\section{Monitor for metabolic changes}

Detecting metabolic effects requires vigilance. Recommendations from the American Diabetes Association and allied organizations in 2004 are outlined in Table $2 .{ }^{20}$ In patients with existing metabolic or cardiovascular disease or in those who gain $5 \%$ or more of their initial weight, alternative medications or closer monitoring is required.

\section{Monitor for movement disorders}

Patients should also be monitored for extrapyramidal side effects (Table 2 ), as some may be irreversible (eg, tardive dyskinesia) or otherwise cause distress (eg, restless legs syndrome,

Always

recommend nonpharmacologic strategies for sleep before giving drugs akathisia). Emergence of abnormal movements is most commonly monitored using the Abnormal Involuntary Movement Scale, ${ }^{46}$ a survey consisting of 12 physical examination findings related to movement disorders. The scale can be employed routinely to detect tardive dyskinesia and monitor its severity over time. Provider familiarity with the scale as well as regular monitoring during follow-up visits is imperative to detect tardive dyskinesia early. ${ }^{46}$

\section{Consider potential for abuse}

Risk of drug abuse should be considered when weighing the risks and benefits of prescribing quetiapine. ${ }^{10}$ It is important to identify patients who are at high risk of drug abuse (eg, prisoners, patients with a history of anxiolytic, sedative, or hypnotic misuse or abuse) at the onset of treatment, and to continue to monitor risk throughout treatment. ${ }^{47} \mathrm{~Pa}$ - tients should be monitored for signs of tolerance, such as increasing dose or seeking other drugs. $^{2}$

However, the risk of misuse, abuse, and dependence with quetiapine is not as high as with benzodiazepines and Z-drugs, which require extensive follow-up and monitoring, possibly including in-person visits every 6 months to 1 year (minimum of every 3 months if the patient is also taking other controlled medications), yearly drug screening, monitoring in a state prescription monitoring and reporting system, limitations on quantity prescribed, and 2-point identification for prescribers, depending on local legislation. ${ }^{33,48}$ Second-generation antipsychotics do not require this level of monitoring.

\section{BOTTOM LINE}

In general, with proper monitoring, quetiapine may help to treat insomnia in patients with comorbid schizophrenia or mood disorders. Some data also support the use of quetiapine for nightmares and insomnia related to PTSD.

Evidence is insufficient to support the broad use of quetiapine to treat insomnia in the general patient population. Several organizations-including the American Diabetes Association, American Psychiatric Association, American Association of Clinical Endocrinologists, North American Association for the Study of Obesity, and the American Geriatric Society - have cautioned against using quetiapine off-label for sleep..$^{20,35}$

Physicians need alternatives when facing difficult decisions about managing highly distressed patients. Other FDA-approved medications for sleep should be tried before quetiapine. And nonpharmacologic strategies, such as meditation, cognitive behavioral therapy for insomnia, and sleep hygiene, should always be recommended before drugs are given.

\section{DISCLOSURES}

The authors report no relevant financial relationships which, in the context of their contributions, could be perceived as a potential conflict of interest. 


\section{REFERENCES}

1. Hálfdánarson Ó, Zoëga H, Aagaard L, et al. International trends in antipsychotic use: a study in 16 countries, 20052014. Eur Neuropsychopharmacol 2017; 27(10):10641076. doi:10.1016/j.euroneuro.2017.07.001

2. Pringsheim T, Gardner DM. Dispensed prescriptions for quetiapine and other second-generation antipsychotics in Canada from 2005 to 2012: a descriptive study. CMAJ Open 2014; 2(4):E225-E232. doi:10.9778/cmajo.20140009

3. US Food and Drug Administration. Highlights of prescribing information: Seroquel. Accessed February 12, 2021. https://www.accessdata.fda.gov/drugsatfda_docs/ label/2020/020639s069lbl.pdf.

4. Sankaranarayanan J, Puumala SE. Antipsychotic use at adult ambulatory care visits by patients with mental health disorders in the United States, 1996-2003: national estimates and associated factors. Clin Ther 2007; 29(4):723-741. doi:10.1016/j.clinthera.2007.04.017

5. Bertisch SM, Herzig SJ, Winkelman JW, Buettner C National use of prescription medications for insomnia: NHANES 1999-2010. Sleep 2014; 37(2):343-349. doi:10.5665/sleep.3410

6. Haw C, Stubbs J. Off-label use of antipsychotics: are we mad? Expert Opin Drug Saf 2007; 6(5):533-545. doi:10.1517/14740338.6.5.533

7. Osborne V, Davies M, Layton D, Shakir SAW. Utilisation of extended release quetiapine (Seroquel $\mathrm{XL}^{\mathrm{TM}}$ ): results from an observational cohort study in England. Eur Psychiatry 2016; 33:61-67. doi:10.1016/j.eurpsy.2015.12.004

8. Huthwaite M, Tucker M, McBain L, Romans S. Off label or on trend: a review of the use of quetiapine in New Zealand. N Z Med J 2018; 131(1474):45-50. pmid:29723178

9. Kim S, Lee G, Kim E, Jung H, Chang J. Quetiapine misuse and abuse: is it an atypical paradigm of drug seeking behavior? J Res Pharm Pract 2017; 6(1):12-15. doi:10.4103/2279-042X.200987

10. Vento AE, Kotzalidis GD, Cacciotti M, et al. Quetiapine abuse fourteen years later: where are we now? A systematic review. Subst Use Misuse 2020; 55(2):304-313. doi:10.1080/10826084.2019.1668013

11. Atkin T, Comai S, Gobbi G. Drugs for insomnia beyond benzodiazepines: pharmacology, clinical applications, and discovery. Pharmacol Rev 2018; 70(2):197-245. doi:10.1124/pr.117.014381

12. Richelson E, Souder T. Binding of antipsychotic drugs to human brain receptors focus on newer generation compounds. Life Sci 2000; 68(1):29-39. doi:10.1016/s0024-3205(00)00911-5

13. Stahl SM. Dopamine system stabilizers, aripiprazole, and the next generation of antipsychotics, part 1, "Goldilocks" actions at dopamine receptors. J Clin Psychiatry 2001; 62(11):841-842. doi:10.4088/jcp.v62n1101

14. Daly EJ, Trivedi MH. A review of quetiapine in combination with antidepressant therapy in patients with depression. Neuropsychiatr Dis Treat 2007; 3(6):855-867. doi:10.2147/ndt.s1862

15. DeVane CL, Nemeroff CB. Clinical pharmacokinetics of quetiapine: an atypical antipsychotic. Clin Pharmacokinet 2001; 40(7):509-522. doi:10.2165/00003088-200140070-00003

16. Roehrs T, Roth T. Insomnia pharmacotherapy. Neurotherapeutics 2012; 9(4):728-738. doi:10.1007/s13311-012-0148-3

17. Maher AR, Theodore G. Summary of the comparative effectiveness review on off-label use of atypical antipsychotics. J Manag Care Pharm 2012; 18(5 suppl B):S1-S20. doi:10.18553/jmcp.2012.18.s5-b.1
18. Leucht S, Cipriani A, Spineli L, et al. Comparative efficacy and tolerability of 15 antipsychotic drugs in schizophrenia: a multiple-treatments meta-analysis. Lancet 2013; 382(9896):951-962. doi:10.1016/S0140-6736(13)60733-3

19. Xu H, Zhuang X. Atypical antipsychotics-induced metabolic syndrome and nonalcoholic fatty liver disease: a critical review. Neuropsychiatr Dis Treat 2019; 15:20872099. doi:10.2147/NDT.S208061

20. American Diabetes Association; American Psychiatric Association; American Association of Clinical Endocrinologists; North American Association for the Study of Obesity. Consensus development conference on antipsychotic drugs and obesity and diabetes. Diabetes Care 2004; 27(2):596-601. doi:10.2337/diacare.27.2.596

21. Solmi M, Murru A, Pacchiarotti I, et al. Safety, tolerability, and risks associated with first- and second-generation antipsychotics: a state-of-the-art clinical review. Ther Clin Risk Manag 2017; 13:757-777. doi:10.2147/TCRM.S11732

22. Cates ME, Jackson CW, Feldman JM, Stimmel AE, Woolley TW. Metabolic consequences of using low-dose que tiapine for insomnia in psychiatric patients. Community Ment Health J 2009; 45(4):251-254. doi:10.1007/s10597-009-9200-0

23. Carr CN, Lopchuk S, Beckman ME, Baugh TB. Evaluation of the use of low-dose quetiapine and the risk of metabolic consequences: a retrospective review. Ment Health Clin 2016; 6(6):308-313. doi:10.9740/mhc.2016.11.308

24. Williams SG, Alinejad NA, Williams JA, Cruess DF. Statistically significant increase in weight caused by low-dose quetiapine. Pharmacotherapy 2010; 30(10):1011-1015. doi:10.1592/phco.30.10.1011

25. Nemeroff CB, Kinkead B, Goldstein J. Quetiapine: preclinical studies, pharmacokinetics, drug interactions, and dosing. J Clin Psychiatry 2002; 63(suppl 13):5-11. pmid:12562141

26. Calabrese JR, Keck PE Jr, Macfadden W, et al. A randomized, double-blind, placebo-controlled trial of quetiapine in the treatment of bipolar I or II depression. Am J Psychiatry 2005; 162(7):1351-1360. doi:10.1176/appi.ajp.162.7.1351

27. Thase ME, Macfadden W, Weisler RH, et al. Efficacy of quetiapine monotherapy in bipolar I and II depression: a double-blind, placebo-controlled study (the BOLDER II study). J Clin Psychopharmacol 2006; 26(6):600-609. doi:10.1097/01.jcp.0000248603.76231.b7

28. Gareri P, Segura-Garcia C, Manfredi VG, et al. Use of atypical antipsychotics in the elderly: a clinical review. Clin Interv Aging 2014; 9:1363-1373. doi:10.2147/CIA.S63942

29. 2019 American Geriatrics Society Beers Criteria Update Expert Panel. American Geriatrics Society 2019 updated AGS Beers Criteria for potentially inappropriate medication use in older adults. J Am Geriatr Soc 2019; 67(4):674694. doi:10.1111/jgs.15767

30. Jin H, Shih PA, Golshan S, et al. Comparison of longerterm safety and effectiveness of 4 atypical antipsychotics in patients over age 40: a trial using equipoise-stratified randomization. J Clin Psychiatry 2013; 74(1):10-18. doi:10.4088/JCP.12m08001

31. American Psychiatric Association. Diagnostic and Statistical Manual of Mental Disorders. 5th ed. Arlington, VA: American Psychiatric Association; 2013.

32. Roth T. Insomnia: definition, prevalence, etiology, and consequences. J Clin Sleep Med 2007; 3(5 suppl):S7-S10. pmid:17824495

33. Parsons G. Dependence on benzodiazepines or Z-drugs: having that conversation. Pharm J 2012; 289:399-402.

34. Cohrs S, Rodenbeck A, Guan Z, et al. Sleep-promoting properties of quetiapine in healthy subjects. Psychophar- 
macology (Berl) 2004; 174(3):421-429. doi:10.1007/s00213-003-1759-5

35. Schutte-Rodin S, Broch L, Buysse D, Dorsey C, Sateia M Clinical guideline for the evaluation and management of chronic insomnia in adults. J Clin Sleep Med 2008; 4(5):487-504. pmid:18853708

36. Wine JN, Sanda C, Caballero J. Effects of quetiapine on sleep in nonpsychiatric and psychiatric conditions. Ann Pharmacother 2009; 43(4):707-713. doi:10.1345/aph.1L320

37. Anderson SL, Vande Griend JP. Quetiapine for insomnia: a review of the literature. Am J Health Syst Pharm 2014; 71(5):394-402. doi:10.2146/ajhp130221

38. McElroy SL, Weisler RH, Chang W, et al. A double-blind, placebo-controlled study of quetiapine and paroxetine as monotherapy in adults with bipolar depression (EMBOLDEN II). J Clin Psychiatry 2010; 71(2):163-174. doi:10.4088/JCP.08m04942gre

39. Endicott J, Paulsson B, Gustafsson U, Schiöler H, Hassan M. Quetiapine monotherapy in the treatment of depressive episodes of bipolar I and II disorder: improvements in quality of life and quality of sleep. J Affect Disord 2008; 111(2-3):306-319. doi:10.1016/j.jad.2008.06.019

40. Robert S, Hamner MB, Kose S, Ulmer HG, Deitsch SE, Lorberbaum JP. Quetiapine improves sleep disturbances in combat veterans with PTSD: sleep data from a prospective, open-label study. J Clin Psychopharmacol 2005; 25(4):387-388. doi:10.1097/01.jcp.0000169624.37819.60

41. Villarreal G, Hamner MB, Cañive JM, et al. Efficacy of quetiapine monotherapy in posttraumatic stress disorder: a randomized, placebo-controlled trial. Am J Psychiatry 2016; 173(12):1205-1212. doi:10.1176/appi.ajp.2016.15070967

42. Byers MG, Allison KM, Wendel CS, Lee JK. Prazosin versus quetiapine for nighttime posttraumatic stress disorder symptoms in veterans: an assessment of long-term comparative effectiveness and safety. J Clin Psychopharmacol 2010; 30(3):225-229. doi:10.1097/JCP.0b013e3181dac52f

43. Tassniyom K, Paholpak S, Tassniyom S, Kiewyoo J. Quetiapine for primary insomnia: a double blind, randomized controlled trial. J Med Assoc Thai 2010; 93(6):729734. pmid:20572379

44. Chow ES, Zangeneh-Kazemi A, Akintan O, Chow-Tung E, Eppel A, Boylan K. Prescribing practices of quetiapine for insomnia at a tertiary care inpatient child and adolescent psychiatry unit: a continuous quality improvement project. J Can Acad Child Adolesc Psychiatry 2017; 26(2):98103. pmid:28747932

45. Kelly M, Dornan T, Pringsheim T. The lesser of two evils: a qualitative study of quetiapine prescribing by family physicians. CMAJ Open 2018; 6(2):E191-E196. doi:10.9778/cmajo.20170145

46. Gharabawi GM, Bossie CA, Lasser RA, Turkoz I, Rodriguez S, Chouinard G. Abnormal Involuntary Movement Scale (AIMS) and Extrapyramidal Symptom Rating Scale (ESRS): cross-scale comparison in assessing tardive dyskinesia. Schizophr Res 2005; 77(2-3):119-128. doi:10.1016/j.schres.2005.03.008

47. Mattson ME, Albright VA, Yoon J, Council CL. Emergency department visits involving misuse and abuse of the antipsychotic quetiapine: results from the Drug Abuse Warning Network (DAWN). Subst Abuse 2015; 9:39-46. doi:10.4137/SART.S22233

48. US Department of Justice; Drug Enforcement Administration, Diversion Control Office. Title 21 United States Code Controlled Substances Act. Accessed February 12, 2021. https://www.deadiversion.usdoj.gov/21cfr/21usc/.

Address: Vania Modesto-Lowe, MD, MPH, Connecticut Valley Hospital, PO Box 351, Silver Street, Middletown, CT 06457; vania.modesto-lowe@ct.gov 